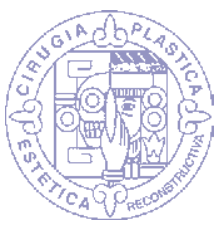

\title{
Complicaciones en pacientes operados de reducción de fracturas naso-órbito-etmoidales de acuerdo con la clasificación de Manson y Markowitz
}

\author{
Complications in patients operated for reduction of naso-orbito-ethmoidal \\ fractures according to the Manson and Markowitz classification \\ Dr. José Roberto Hernández-Méndez,* Dr. Jacobo Felemovicius-Hermangus, ${ }^{*}$ \\ Dr. Federico Íñigo-Arroyo, ${ }^{\S}$ Dr. Emanuel Arceo-Martínez,** \\ Dr. Roy Ismael Villafranca-Andino*
}

Palabras clave: Fractura NOE, fractura naso-órbito-etmoidal, ortognática, trauma facial.

Keywords: NOE fracture, naso-orbital ethmoid fracture, orthognathic, facial trauma.

\footnotetext{
* Médico residente de Cirugía Plástica y Reconstructiva. ¥ Cirujano plástico adscrito a la División de Ortognática y Trauma Facial.

$\S$ Fellowship de Cirugía Ortognática y Trauma Facial.
}

Servicio de Cirugía Plástica, Estética y Reconstructiva. Hospital General «Dr. Manuel Gea González».

\section{RESUMEN}

Describimos las complicaciones ocurridas en los pacientes que fueron operados de reducción de fracturas naso-órbitoetmoidales clasificadas según Manson y Markowitz, en el Servicio de Cirugía Plástica y Reconstructiva del Hospital General «Dr. Manuel Gea González». Realizamos un estudio descriptivo, observacional, transversal y retrospectivo en el que revisamos los expedientes clínicos en la base de datos de la División de Ortognática del Servicio de Cirugía Plástica y Reconstructiva del Hospital General «Dr. Manuel Gea González»; éste incluyó pacientes con diagnóstico de fractura naso-órbito-etmoidal (NOE) dentro del periodo del 1 de enero de 2010 al 31 de diciembre de 2018, incluyendo aquéllos que fueron sometidos a reducción abierta con fijación interna. Identificamos y registramos 16 pacientes (37.2\%) con secuelas posteriores al manejo de este tipo de fractura, y encontramos distopia cantal $(9.3 \%, n=4)$, enoftalmos $(6.9 \%, \mathrm{n}=3)$ y telecanto $(6.0 \%, \mathrm{n}=3)$. Se registró deformidad nasal en silla de montar $(2.3 \%, \mathrm{n}=1)$, ectropión $(2.3 \%, \mathrm{n}=1)$ y parestesia malar $(6.9 \%, \mathrm{n}=3)$. El $8.8 \%$ de las fracturas tipo I tuvieron complicaciones; las fracturas tipo II presentaron un $72.7 \%$ de complicaciones, principalmente distopia cantal $(n=2)$, y hubo reporte de complicaciones en todas las fracturas tipo III. Concluimos que existe un mayor porcentaje de complicaciones en pacientes con fracturas de presentación más compleja.

\section{ABSTRACT}

We describe the complications in patients operated for reduction of naso-orbital ethmoid fractures according to the classification of Manson and Markowitz in the Plastic and Reconstructive Surgery Service of the Dr. Manuel Gea González General Hospital. We conducted a descriptive, observational, retrospective cross-sectional study, in which we reviewed the clinical records in the database of the orthognathic division of the plastic and reconstructive surgery of the Dr. Manuel Gea González Hospital, which included patients with a diagnosis of nasoorbital ethmoid (NOE) fracture in the period from January 1, 2010 to December 31, 2018, including those subject to open reduction with internal fixation. We identified and registered 16 patients (37.2\%) with sequelae after the management of this type of fracture, finding cantal dystopia (9.3\%, $n=4)$, enophthalmos $(6.9 \%, n=3)$ and telecanthus $(6.0 \%, n=3)$. Saddle nasal deformity $(2.3 \%$, $n=1)$, ectropion $(2.3 \%, n=1)$ and malar paresthesia $(6.9 \%, n=3)$ were recorded. $8.8 \%$ of type I fractures had complications; type II fractures presented $72.7 \%$ of complications, mainly cantal dystopia $(n=2)$ and there were reports of complications in all type III fractures. We conclude that there is a higher percentage of complications in patients with more complex presentation fractures.

\section{INTRODUCCIÓN}

T as fracturas naso-órbito-etmoidales (NOE) $\perp$ representan menos de un $5 \%$ de las fracturas faciales en adultos y menos del $15 \%$ en niños. ${ }^{1}$ Entre las causas principales de este tipo de lesiones figuran los accidentes automovilísticos y las agresiones por terceros. Se estima que hasta el $60 \%$ de las fracturas NOE se asocian con fracturas órbito-cigomáticas y aproximadamente el $20 \%$ se diagnostica en el contexto de fracturas panfaciales. A pesar de 
Los autores de este artículo no tienen conflicto de intereses qué declarar.

Recibido:

05 septiembre 2019 Aceptado para publicar: 04 noviembre 2019 su baja incidencia, éstas se encuentran entre las fracturas cráneo-maxilofaciales más desafiantes de diagnosticar y tratar, y su tratamiento inadecuado impacta significativamente en el resultado estético y funcional. ${ }^{2-5} \mathrm{En}$ una fractura NOE están involucrados los huesos de la pared nasal lateral, proceso frontal del hueso maxilar, la lámina papirácea etmoidal de la pared orbitaria medial, el reborde orbitario inferior y el contrafuerte naso-maxilar. ${ }^{6,7}$

El paciente, a menudo, presenta hemorragia subconjuntival, equimosis y edema en el área cantal medial, además de dolor y crepitación en la palpación. La nariz se puede retruir e impactar (telescopar) en el área de la sutura nasofrontal, con falta de soporte para el tabique nasal y cartílagos. ${ }^{8}$ Cuando no hay definición de la anatomía ósea en el área cantal medial y la posible separación lateral del canto medial, con mayor distancia intercantal (telecanto), se requieren pruebas positivas de inestabilidad cantal, como la tracción bimanual (que consiste en colocar un instrumento en la nariz y empujar lateralmente en el área cantal medial para evaluar la inestabilidad y la crepitación, lo que sugiere una fractura inestable por NOE) y la cuerda de arco (que consiste en que el cirujano pueda ser capaz de tomar el párpado o usar unas pinzas para asir la piel en el área cantal medial y tirar de él lateralmente, también llamada «prueba de cuerda de arco». La falta de resistencia o movimiento del hueso subyacente es indicativa de una fractura), así como desnivel en los bodes orbitarios. ${ }^{9-11}$ Es necesario descartar fugas de líquido cefalorraquídeo en fracturas desplazadas bilaterales para minimizar el riesgo de meningitis temprana o tardía, y descartar lesiones en los globos y aparato lagrimal. ${ }^{11}$

Markowitz, Manson y colaboradores, en 1991, clasificaron las fracturas en:

- Tipo I. Fractura formada por un solo fragmento óseo grande con el tendón cantal medial intacto

- Tipo II. Fractura conminuta con el tendón cantal medial insertado

- Tipo III. Tendón cantal medial avulsionado de la fosa lagrimal y del fragmento óseo

Éstas pueden ser lesiones unilaterales o bilaterales. $^{12}$
A menudo, en relación con los hallazgos radiográficos, se puede distinguir entre una fractura NOE tipo I y una de tipo II / III por el grado de conminución observado en la tomografía computarizada (TC). Una TC-3D puede ayudar a evaluar la gravedad de la lesión, pero el diagnóstico final requiere hallazgos clínicos y un tendón cantal medial avulsionado a partir de su inserción en el hueso, lo cual, frecuentemente, se tiene que determinar de forma transoperatoria. ${ }^{13}$

Se indica la realización de cirugía de la fractura NOE para corregir el telecanto, acortar la fisura palpebral, liberar la obstrucción de la vía aérea y mejorar el contorno del dorso nasal y de tejidos blandos. El acceso quirúrgico se realiza mediante la exposición de la región por medio de incisiones coronal, glabelar, palpebral inferior y/o vestibular. En algunos casos, se puede tener acceso por heridas ya existentes. ${ }^{14,15} \mathrm{La}$ parte más importante es la reducción transnasal de la pared medial de los bordes orbitarios. La reducción abierta y la fijación interna para estabilizar el fragmento central y el tendón cantal medial producen resultados superiores a la reducción cerrada. ${ }^{10}$

El tratamiento en las fracturas NOE tipo I puede ser conservador en aquellas fracturas no desplazadas o mínimas, esto es, sin telecanto, sin embargo, es necesario su seguimiento estricto. En la reducción abierta con fijación interna, el objetivo es la reducción del proceso frontal del maxilar y el reposicionamiento del tendón cantal medial; la fijación interna para esta fractura puede incluir 1, 2 o 3 puntos. La primera placa, generalmente, se coloca en una posición inferior desde el fragmento fracturado hasta el hueso maxilar de la abertura piriforme. Si se necesita una fijación de dos puntos, la segunda placa se coloca en la sutura frontonasal y una tercera placa puede ser necesaria en caso de fracturas asociadas. ${ }^{8,9}$

El manejo de las fracturas tipo II incluye la reducción abierta con fijación interna, ya que el fragmento de hueso portador cantal requiere un reposicionamiento exacto, lo que restaura la distancia intercantal. Cuando existe afectación del hueso nasal puede ser necesaria la fijación con placas. Si el dorso de la nariz está triturado o si no se puede reducir completamente, se realiza una reconstrucción con un injerto 
de hueso. En aquellos casos de fractura NOE bilateral con afectación del hueso nasal, se debe considerar una sobrecorrección de la distancia intercantal (normal: hombres de 33 a $34 \mathrm{~mm}$ y mujeres de 32 a $33 \mathrm{~mm}$ ). La fijación transnasal incluye el uso de placas, tornillos, malla o alambre. Se necesitan como mínimo tres puntos de fijación: hueso frontal, reborde orbitario y abertura piriforme, en este orden. 8,9

Las fracturas NOE tipo III requieren reducción abierta con fijación interna; el dorso nasal casi siempre está fracturado en forma extensa, al igual que el proceso frontal del maxilar. El tendón cantal medial requiere cantopexia. Si el dorso nasal no se puede reducir completamente, se realiza una reconstrucción con un injerto óseo laminado. Se necesita un mínimo de tres puntos de fijación: hueso frontal, reborde orbitario y abertura piriforme, en este orden; además, pueden ser necesarias placas adicionales. Es importante señalar la importancia de la reconstrucción nasal primaria en todas las fracturas NOE. ${ }^{8,9}$ Las fracturas NOE a menudo se asocian con otras fracturas faciales. ${ }^{15}$

La fractura NOE pediátrica es una presentación desafiante entre todas las fracturas maxilofaciales. La fijación rígida ha demostrado que resulta en la restricción del crecimiento en modelos animales. Las técnicas que utilizan sistemas de placas absorbibles se emplean comúnmente para cirugía craneofacial pediátrica, lo que evita la posible necesidad de retirar la placa, aunque ningún estudio documenta el uso de esta técnica en las fracturas NOE pediátricas. ${ }^{16}$ A pesar de la incidencia potencialmente mayor de obstrucción del conducto naso-lagrimal que causa epífora, la fijación transnasal sigue siendo la modalidad de tratamiento de elección para la estabilización cantal medial en fracturas NOE tipo II y tipo III en pacientes pdiátricos. ${ }^{5}$

Las fracturas de la región naso-órbito-etmoidal causan importantes defectos funcionales y estéticos. ${ }^{17}$ La mayoría de las complicaciones asociadas con las fracturas NOE son resultado de un diagnóstico o tratamiento inadecuado en el momento de la lesión inicial. Dentro de las complicaciones postoperatorias descritas, las series reportan porcentajes variables de presentación tanto en frecuencia como en gravedad, dependiendo de los centros de atención y experiencia de los mismos; destacan en la región nasal: deformidad estética, obstrucción y desviación nasal; en la región orbitaria: telecanto, enoftalmos, diplopía, pérdida o disminución de la agudeza visual y ptosis palpebral; en la región etmoidal: defectos craneales, fistulas de líquido cefalorraquídeo y otras como infecciones (celulitis orbitaria o frontal, meningitis), sinusitis fronto-etmoidal, ruptura de material de osteosíntesis, necrosis de piel en región cantal medial, e incluso la muerte. ${ }^{18}$

\section{MATERIAL Y MÉTODO}

Realizamos un estudio descriptivo, observacional, transversal y retrospectivo. Revisamos los expedientes clínicos de pacientes con diagnóstico de fracturas naso-órbito-etmoidales (NOE) en la base de datos de la división de ortognática en Servicio de Cirugía Plástica y Reconstructiva del Hospital General «Dr. Manuel Gea González», en el periodo del 1 de enero de 2010 al 31 de diciembre de 2018. Se incluyeron pacientes que fueron sometidos a reducción abierta con fijación interna y se excluyeron los que no contaban con registro de seguimiento después del manejo quirúrgico. Se registró la presencia de complicaciones postoperatorias en pacientes sometidos a reducción abierta y fijación interna de fracturas naso-órbito-etmoidales, de acuerdo con la clasificación de Manson y Markowitz y se utilizó estadística descriptiva para reportar los resultados utilizando medidas de tendencia central.

\section{RESULTADOS}

De los 754 casos de fracturas faciales registradas en el periodo comprendido entre enero de 2010 y diciembre de 2018, se identificaron 48 fracturas tipo NOE sometidas a reducción abierta y fijación interna en 43 pacientes, alcanzando una incidencia de $6.3 \%$ en 8 años.

El 90.6\% de los casos correspondieron al sexo masculino. La mediana de edad fue de 33 años, con mínima de 17 y máxima de 87 (Tabla 1). La lateralidad en estas lesiones fue de predominio izquierdo en 29 pacientes (67.4\%), seguida del derecho en $9(20.9 \%)$ y bilateral en 5 (11.6\%).

Tomando como referencia la clasificación de Manson y Markowitz, con base en la localización y lesión del ligamento cantal interno, el tipo de 
Tabla 1: Características demográficas de $\mathbf{4 3}$ pacientes.

Características demográficas

\begin{tabular}{lcc}
\hline Sexo & $\begin{array}{c}\text { Hombres } \\
(\mathrm{n}=39)\end{array}$ & $\begin{array}{c}\text { Mujeres } \\
(\mathrm{n}=4)\end{array}$ \\
Edad (años) & $33(17-87)$ & $34(32-38)$ \\
Mediana (m-M) & & \\
\hline
\end{tabular}

fractura predominante correspondió a la fractura tipo I con un $70.8 \%(n=34)$, tipo II con $22.9 \%$ $(\mathrm{n}=11)$ y tipo III con $6.3 \%(\mathrm{n}=3)$.

La asociación con lesiones óseas o de tejidos blandos registró las siguientes 24 fracturas asociadas (55.8\%): órbito-cigomáticas (54.1\%, $\mathrm{n}=13)$, órbita, piso y pared lateral $(16.6 \%$, $\mathrm{n}=4)$, maxilar $(12.5 \%, \mathrm{n}=3)$, frontal $(8.3 \%$, $\mathrm{n}=2)$ y mandibular $(8.3 \%, \mathrm{n}=2)$.

Las lesiones en tejidos blandos asociadas se presentaron en 13 casos (30.2\%). En primer lugar, las heridas abiertas fueron $(n=8)$ hematoma subdural $(n=1)$, uveítis $(n=2)$, desprendimiento de retina $(n=1)$ y trauma ocular abierto $(n=1)$.

Los accesos quirúrgicos empleados en estos pacientes correspondieron al acceso transconjuntival más vestibular superior $(69.7 \%$, $\mathrm{n}=30$ ), glabelar extendido más vestibular superior $(13.9 \%, \mathrm{n}=6)$, acceso en heridas previas $(11.6 \%, n=5)$ y coronal $(4.6 \%, n=2)$.

En los casos en los que fue necesaria la reconstrucción de órbita $(25.5 \%, \mathrm{n}=11)$, se utilizó principalmente injerto de cartílago $(11.6 \%, \mathrm{n}=5)$. Se identificaron y registraron 16 pacientes $(37.2 \%)$ con las siguientes secuelas posteriores al manejo de estas fracturas: distopia cantal $(9.3 \%, n=4)$, enoftalmos $(6.9 \%, n=3)$ y telecanto $(6.0 \%, n=3)$; además registro de deformidad nasal en silla de montar $(2.3 \%$, $\mathrm{n}=1)$, ectropión $(2.3 \%, \mathrm{n}=1)$ y parestesia malar $(9.3 \%, n=4)$. El $8.8 \%$ de las fracturas tipo I tuvieron complicaciones; las fracturas tipo II presentaron $72.7 \%$ de complicaciones, principalmente distopia cantal $(n=2)$ y se registró complicaciones en todas las fracturas tipo III (Tabla 2).

\section{DISCUSIÓN}

Acorde con los resultados de Kelley de 2005, en un análisis retrospectivo sobre 294 fracturas en un centro de trauma, las fracturas tipo NOE presentaron una incidencia menor al 5 y $15 \%$ de las fracturas faciales en adultos y niños, respectivamente. ${ }^{19}$ En nuestro Servicio, las fracturas tipo NOE representan un $6.7 \%$ de las fracturas faciales en adultos en este reporte de 8 años. Nguyen, en 2010, ${ }^{4}$ y Etemadi, en $2017,{ }^{5}$ reportan las fracturas tipo I como las más frecuentes en sus estudios retrospectivos, sin embargo, en nuestra revisión encontramos un $70.8 \%$. Las fracturas órbito-cigomáticas se han asociado hasta en un $60 \%$ de los casos (Baril, 2013) ${ }^{11}$ y nosotros encontramos una presentación del 54\%.

Ferreira Brasileiro, en 2006, en su estu-

Tabla 2: Complicaciones postquirúrgicas.

\begin{tabular}{lcccc} 
& & \multicolumn{3}{c}{ Tipos de fracturas } \\
\cline { 3 - 5 } & $\begin{array}{c}\text { Núm. de } \\
\text { complicaciones }\end{array}$ & $\begin{array}{c}\text { Tipo I } \\
(\mathrm{n}=34)\end{array}$ & $\begin{array}{c}\text { Tipo II } \\
(\mathrm{n}=11)\end{array}$ & $\begin{array}{c}\text { Tipo III } \\
(\mathrm{n}=3)\end{array}$ \\
\hline Distopia cantal & 4 & 1 & 2 & 1 \\
Enoftalmos & 3 & 1 & 1 & 1 \\
Telecanto & 3 & - & 2 & 1 \\
Deformidad nasal & 1 & - & - & 1 \\
Ectropión & 1 & - & 1 & - \\
Parestesia malar & 4 & 1 & 2 & $5(116 \%)$ \\
Total & 16 & $3(8.8 \%)$ & $8(72.7 \%)$ & \\
\hline
\end{tabular}


dio «Epidemiological analysis of maxillofacial fractures in Brazil: a 5-year prospective study» demostró, con 1,024 casos de fracturas faciales, que las fracturas NOE presentan la mayor tasa de complicaciones de todas las fracturas faciales. ${ }^{7}$ Kelley, en 2005, reportó una tasa global de complicaciones del 7.8\%. ${ }^{19}$ Algunos reportes abarcan valores desde el $34 \%$ hasta el 100\%, (como los de Ferreira en 2006 y Reiter en 2017), 7,10 a pesar de que el nuestro es de un $37.2 \%$, no consideramos inconformidades estéticas. No encontramos en nuestros registros complicaciones reportadas en otras series, como epífora, postcirugía y lesiones temporales del conducto naso-lagrimal, así como infecciones postratamiento quirúrgico. ${ }^{20}$

Las deformidades características relacionadas con las fracturas NOE tratadas inadecuadamente incluyen una nariz acortada y retruida, fisura palpebral acortada, telecanto, enoftalmos y diplopía. ${ }^{21,22}$

Una de las complicaciones más comunes derivadas de las fracturas NOE es el telecanto traumático, con una incidencia tan alta como del $70 \%$, según lo reportado por Cruse, ${ }^{21}$ en 1990 y Merk, ${ }^{22}$ en 1995, en sus respectivos estudios retrospectivos, debido a la lesión y avulsión del ligamento cantal medial. ${ }^{17,23}$ Markowitz y su equipo, en su artículo sobre el manejo del tendón cantal medial en fracturas $\mathrm{NOE}$, encontraron que sólo el 3\% de los pacientes que sufrieron fracturas NOE con lesiones contundentes tuvieron desplazamiento cantal del tendón. ${ }^{12}$ Otros estudios han mostrado mayores proporciones de desplazamiento cantal, pero éstas pueden estar relacionadas con la disección quirúrgica y no con la lesión. Otra imperfección estética asociada con las fracturas NOE, tal como comenta Vora, ${ }^{17}$ en 2000 y Potter, ${ }^{23}$ en 2006, incluyen deformidades nasales debido a la pérdida de soporte de la nariz. Sus escritos tienen como objetivo la presentación de un algoritmo de manejo exclusivo de deformidad nasal como secuela en este tipo de pacientes.

A partir de los resultados descritos por Pfeifer, ${ }^{24}$ en 1941, quien incluyó el análisis de 120 casos de fracturas con compromiso orbitario, se sabe que el enoftalmos se presenta en más del $50 \%$ de los pacientes. ${ }^{25} \mathrm{La}$ retrusión del tercio medio facial se produce como resultado del telescopaje de los huesos nasales hacia adentro de la zona de deformación, como lo describió Rebeca Fraioli y colaboradores, en $2008 .^{26}$ La epífora puede ocurrir como resultado de una obstrucción del sistema naso-lagrimal, o por una mala posición postoperatoria del párpado; ${ }^{27,28}$ ésta tiene una prevalencia del 47\% (Gruss, ${ }^{15}$ 1985: análisis de 46 casos; Becelli, ${ }^{27}$ 2005: análisis de 58 casos). La diplopía puede ser otra secuela de fracturas tipo NOE y puede ocurrir debido al desplazamiento lateral de los fragmentos de la pared orbital medial hacia la órbita o el desplazamiento medial del hueso etmoidal, tal como lo demostró Lamb, en 1990, después de la revisión de los resultados de 33 pacientes que presentaron trauma facial, mediante TC, de los cuales el $51 \%$ correspondió a fracturas tipo NOE, encontrando diplopía por desplazamiento lateral y medial en el 35 y $12 \%$ respectivamente. ${ }^{29-31}$ También pueden ocurrir deficiencias visuales y ceguera. ${ }^{20,32-34}$ Ansari, en su estudio retrospectivo de 19 años, informó sólo un caso de ceguera entre 19 pacientes con fracturas $\mathrm{NOE}^{33}$ que es el tipo de fractura facial que involucra menos lesiones oculares. En un estudio de revisión realizado por Bossert y Girotto, ${ }^{34}$ la incidencia de ceguera relacionada con fracturas faciales se reportó en alrededor del $3 \% .^{32-36}$ La lesión cerebral puede ser una consecuencia mortal de las fractura $\mathrm{NOE}$, que se produce cuando los fragmentos óseos penetran en la fosa craneal anterior. ${ }^{31}$ La rinorrea de LCR también puede ocurrir traumáticamente debido a fracturas tipo NOE, aproximadamente en el $12 \%$ de los casos, como lo demostró Bell, en 2004, en su análisis retrospectivo de 735 casos. ${ }^{35}$

La infección concomitante se informa rara vez en las fracturas NOE. Kyrgidis y su equipo, en un estudio de 1,239 casos de fracturas maxilofaciales, informaron siete casos de infección en fracturas NOE junto con fracturas panfaciales. ${ }^{36}$ Las secuelas de estas fracturas, conocidas a largo plazo, incluyen principalmente las óseas como: hipertelorismo, retrusión de la parte media de la cara, deformidad nasal (proyección, asimetría); de tejidos blandos: telecanto, enoftalmos, ectropión, engrosamiento y contractura de tejidos blandos y funcionales; epífora, ceguera, anosmia y obstrucción del 
conducto naso-lagrimal. ${ }^{5}$

Aunque el acceso y manejo quirúrgico ha permanecido sin alteraciones en los últimos años, la mejoría en la instrumentación quirúrgica y los procesos de planificación quirúrgica computarizada, como escáner pre y transoperatorio, han reducido las secuelas y han mejorado los resultados y tiempos quirúrgicos. ${ }^{5}$

\section{CONCLUSIONES}

Se ha reportado la presentación escasa de este tipo de fracturas, así como la frecuencia de complejidad en el manejo de las mismas. En este estudio describimos el tipo de complicaciones postratamiento quirúrgico y la presentación de las mismas de acuerdo con el grado de compromiso óseo. Los resultados muestran un mayor porcentaje de complicaciones en los pacientes, en los grupos con presentación más compleja de fractura.

Se requieren estudios analíticos y prospectivos para validar esta relación, de forma que se permita ofrecer información de posibles complicaciones en forma preoperatoria.

\section{REFERENCIAS}

1. Eom T. Analysis of symptoms according to areas of orbital floor in orbital inferior wall fractures. J Craniofac Surg 2015; 26 (3): 647-659.

2. Herford AS. Secondary Treatment of Naso-orbital Ethmoid Injuries. Facial Plast Surg 2017; 33 (6): 591-597.

3. Louis M. Midface Fractures I. Sem Plast Surg 2017; 31 (2): 85-93.

4. Nguyen M. Pearls of naso-orbito-ethmoid trauma. Sem Plast Surg 2010; 24 (4): 23-28.

5. Etemadi M. Management of naso-orbito-ethmoid fractures: a 10 years review. Trauma Mon 2017: 22 (3): 27-30.

6. Martins C, Costa e Silva IE, Campero A et al. Microsurgical Anatomy of the Orbit: The Rule of Seven. Anat Res Int 2011; 2011: 468727. DOI: 10.1155/2011/468727.

7. Ferreira Brasileiro B. Epidemiological analysis of maxillofacial fractures in Brazil: a 5-year prospective study. Oral Surg Oral Med Oral Pathol Oral Radiol Endod 2006; 102 (1): 28-34.

8. Chaudhry O, Isakson M, Franklin A, Maqusi S, El Amm C. Facial Fractures. Plast Reconstr Surg 2018; 141 (5): 742e-758e.

9. Cornelius CP, Gellrich N, Hillerup S, Kusumoto K, Schubert W, Fusetti S. AO Surgery Reference [Internet] Davos: AO Foundation. Available from: https://www2. aofoundation.org/wps/portal/surgery.

10. Reiter MJ. Postoperative CT of the Midfacial Skeleton after Trauma: Review of Normal Appearances and Common Complications. Am J Roentgenol 2017; 209
(4): W238-W248.

11. Barril SE. Naso-orbito-ethmoidal (NOE) fractures: A review. Int Ophthalmol Clin 2013; 53 (4): 149-155.

12. Markowitz BL, Manson PN, Sargent L et al. Management of the Medial Canthal Tendon in Nasoethmoid Orbital Fractures: The Importance of the Central Fragment in Classification and Treatment. Plast Reconstr Surg 1991; 87 (5): 843-853.

13. Hopper RA. Diagnosis of Midface Fractures with CT: What the Surgeon Needs to Know. Radiographics 2006; 26 (3): 783-793.

14. Leipzinger LS. Nasoethmoid orbital fractures. Current concepts and management principles. Clin Plast Surg 1992; 19: 167-93.

15. Gruss JS. Naso-ethmoid-orbital fractures: classification and role of primary bone grafting Plast Reconstr Surg 1985; 75 (3): 303-317.

16. Rosenberger E. Management of nasoethmoid fractures. Curr Opin Otolaryngol Head Neck Surg 2013; 21 (4): 410-6.

17. Vora N. Management of the central nasal support complex in naso-orbital ethmoid fractures. Facial Plast Surg 2000; 16 (2): 181-91.

18. Ellis E. Sequencing treatment for naso-orbito-ethmoid fractures. J Oral Maxillofac Surg 1993; 51 (5): 543-558.

19. Kelley P. Two hundred ninety-four consecutive facial fractures in an urban trauma center: lessons learned. Plast Reconstr Surg 2005; 116 (3): 42e-49e.

20. Mehravaran R, Akbarian G, Nezhad CM et al. Evaluation of the relationship between the pattern of midfacial fractures and amaurosis in patients with facial trauma. J Oral Maxillofac Surg 2013; 71 (6): 1059-62.

21. Cruse CW. Naso-ethmoid-orbital fractures. J Trauma 1990; 20 (7): 551-6.

22. Merkx MA. Effectiveness of primary correction of traumatic telecanthus. Int J Oral Maxillofac Surg 1995; 24 (5): 344-7.

23. Potter JK. Aesthetic management of the nasal component of naso-orbital ethmoid fractures. Plast Reconstr Surg 2006; 117 (1): 10e-8e.

24. Pfeiffer RL. Enophthalmos and diplopia in fractures of the orbital floor. Trans Am Ophthalmol 1941; 39: 492.

25. Baril SE. Naso-orbito-ethmoidal (NOE) fractures: a review. Int Ophthalmol Clin 2013; 53 (4): 149-55.

26. Fraioli RE. Facial fractures: beyond Le Fort. Otolaryngol Clin North Am 2008; 41 (1): 51-76.

27. Becelli R. Posttraumatic obstruction of lacrimal pathways: a retrospective analysis of 58 consecutive naso-orbitoethmoid fractures. J Craniofac Surg 2004; 15 (1): 29-33.

28. Gruss JS. The pattern and incidence of nasolacrimal injury in naso-orbital-ethmoid fractures: the role of delayed assessment and dacryocystorhinostomy. $\mathrm{Br} J$ Plast Surg 1985; 38 (1): 116-21.

29. Dingman RO. Management of injuries of the nasoorbital complex. Arch Surg 1969; 98 (5): 566-71.

30. Stranc MF. The pattern of lacrimal injuries in nasoethmoid fractures. Br J Plast Surg 1970; 23 (4): 339-46.

31. Daly BD. Thin section computed tomography in the evaluation of naso-ethmoidal trauma. Clin Radiol 1990; 41 (4): 272-5.

32. MacKinnon CA. Blindness and severe visual impairment in facial fractures: an 11 year review. Br J Plast Surg 
2002; 55 (1): 1-7.

33. Ansari MH. Blindness after facial fractures: a 19-year retrospective study. J Oral Maxillofac Surg 2005; 63 (2): 229-37.

34. Bossert RP. Blindness following facial fracture: treatment modalities and outcomes. Craniomaxillofac Trauma Reconstr 2009; 2 (3): 117-24.

35. Bell RB. Management of cerebrospinal fluid leak associated with craniomaxillofacial trauma. J Oral Maxillofac Surg 2004; 62 (6): 676-84.

36. Kyrgidis A. Incidence, etiology, treatment outcome and complications of maxillofacial fractures. A retrospective study from Northern Greece. J Craniomaxillofac Surg 2013; 41 (7): 637-43.

\section{Correspondencia:}

Dr. José Roberto Hernández Méndez

Calzada de Tlalpan No. 4585,

Departamento 9. Colonia Toriello Guerra,

14050, Ciudad de México, México.

E-mail: jrhmendez@gmail.com 